\title{
Connectivity interplays with age in shaping contagion over networks with vital dynamics
}

\author{
Carlo Piccardi, Alessandro Colombo, and Renato Casagrandi* \\ Department of Electronics, Information and Bioengineering, Politecnico di Milano, 20133 Milano, Italy
}

(Received 16 June 2014; published 17 February 2015)

\begin{abstract}
The effects of network topology on the emergence and persistence of infectious diseases have been broadly explored in recent years. However, the influence of the vital dynamics of the hosts (i.e., birth-death processes) on the network structure, and their effects on the pattern of epidemics, have received less attention in the scientific community. Here, we study Susceptible-Infected-Recovered(-Susceptible) [SIR(S)] contact processes in standard networks (of Erdös-Rényi and Barabási-Albert type) that are subject to host demography. Accounting for the vital dynamics of hosts is far from trivial, and it causes the scale-free networks to lose their characteristic fat-tailed degree distribution. We introduce a broad class of models that integrate the birth and death of individuals (nodes) with the simplest mechanisms of infection and recovery, thus generating age-degree structured networks of hosts that interact in a complex manner. In our models, the epidemiological state of each individual may depend both on the number of contacts (which changes through time because of the birth-death process) and on its age, paving the way for a possible age-dependent description of contagion and recovery processes. We study how the proportion of infected individuals scales with the number of contacts among them. Rather unexpectedly, we discover that the result of highly connected individuals at the highest risk of infection is not as general as commonly believed. In infections that confer permanent immunity to individuals of vital populations (SIR processes), the nodes that are most likely to be infected are those with intermediate degrees. Our age-degree structured models allow such findings to be deeply analyzed and interpreted, and they may aid in the development of effective prevention policies.
\end{abstract}

DOI: 10.1103/PhysRevE.91.022809

PACS number(s): 89.75.Fb, 87.10.Mn

\section{INTRODUCTION}

Epidemiology is no doubt one of the most successful fields of application of complex network science. It is in fact quite natural to recognize that individuals cannot be treated "as average" in terms of pathogen transmissions. For example, in sexually transmitted or in childhood diseases, the social behavior of each human host varies her risk of infection, thus it can enhance or reduce her role as a potential spreader in the population. A similar heterogeneity emerges in animal populations, where some nodes - such as the older individuals in rodents [1] or the nursery swine farms in Ontario [2] — can play key roles in the spread of infections. This is why the extensive use of ordinary differential equation (ODE) approaches to epidemics, rooted in the pioneering work by Kermack and McKendrick [3], is currently facing a deep revision in light of the complex networks paradigm.

Particularly studied in this context is the problem of disease persistence [4,5], because of its crucial role in public health policies. Being able to identify which individuals are at the highest risk of infection is in fact a priority for health systems, and it can benefit much from insight into the dynamics of contagions [6,7]. While the spreading of diseases over temporal networks [8] or adaptive networks [9] has been studied in recent years, the mechanisms by which birth and death processes can alter the expected outcomes of simple diseases spreading over an otherwise static but heterogeneous network have been mostly ignored. This contrasts with the fact that many of the results on SIR-like epidemic ODE

\footnotetext{
*Author to whom all correspondence should be addressed: renato.casagrandi@polimi.it
}

models with varying total population size [10] focus instead on diseases, such as tuberculosis, where the latent period of exposed individuals is so long that the hosts' demography cannot be ignored $[11,12]$.

The issue of whether one should consider a system open, i.e., subject to demographic variations, is strictly related to the time scales of disease transmission, the time spent by individuals in the infected compartment (the so called infectious period), and the temporal window over which the disease dynamics is observed. For instance, analyzing the spreading of one epidemic wave of plague on the island of Bombay [3] or a single wave of influenza [13] requires different models than those needed to study the long-term patterns. Even for diseases characterized by fast cycles (such as influenza), the introduction of demographic dynamics into the epidemiological model is crucial to investigate the temporal characteristics of the persistent disease in the long run [14].

The recruitment of susceptible individuals that keep diseases at endemic equilibrium is in many cases due to births (think of measles as a paradigmatic example [15]). However, since the SIS model offers a simple way of simultaneously (i) replenishing the susceptible compartment, and (ii) keeping the network size and structure constant throughout time, it has been taken as the core mechanism in the great majority of published studies. As brilliantly synthesized by Nåsell [16], "it turns out that SIR without demography lead to epidemic infections, while both SIR models with demography and SIS models, with or without demography, are associated with endemic infections." Despite the similarity of their dynamical outputs in compartmental models, here we show that SIR and SI(R)S models subject to demography over networks mainly affect individuals with different 
degrees, thus providing quantitatively and qualitatively different results.

Understanding the effects of demography on the existence of epidemic thresholds in epidemiological models is surely important. However, except for a few notable examples [1719], research on this topic has been scarce. In contrast with commonly used epidemiological models on networks, we therefore account for the hosts' demography. This allows us to study the long-term characteristics of epidemics when the vital dynamics of hosts cannot be considered as frozen during the period of interest. Also, to disentangle the underlying causes that generate the degree distributions of infecteds as found below, we investigate the relationships between the age of individuals, their degree, and their epidemiological state via an ad hoc model. This is described and analyzed in the next sections, prior to some concluding remarks.

\section{NETWORK TOPOLOGIES UNDER BIRTH-DEATH PROCESSES}

We model the population as a time-varying network with $N(t)$ nodes (i.e., individuals). We use $k_{i}(t) \geqslant 0$ for the current node degree, i.e., the number of links connecting the $i$ th node. The network is characterized by its degree distribution, and we name $0 \leqslant p_{k}(t) \leqslant 1$ the fraction of nodes having degree $k=0,1, \ldots, \bar{k}$ at time $t(\bar{k}<\infty$ denotes the maximum degree value), therefore $\sum_{k} p_{k}(t)=1$ for all $t$ 's. The possibly time-varying average degree of the network is denoted by $\langle k\rangle=\sum_{k} k p_{k}(t)$. We assume that, during a short time interval $\Delta$, an existing node can die, together with all links departing from it, with probability $\mu \Delta$, irrespective of its degree and its current epidemic state (i.e., no virulence). Such a death process can in principle be more elaborate, but we want to keep demography as simple as possible to minimize the potential sources of dynamical complexity. We therefore assume that each node gives birth to another node with probability $\mu \Delta$, independently of its epidemic state. Newborns attach to existing nodes according to the topology-dependent rules detailed below. Since the natality and mortality rates are identical, the birth-death process is neither biased toward population growth nor extinction, and the network size $N(t)$ is expected to stochastically fluctuate around a constant value. Although the demographic process does not change the average number of nodes in the network, it is interesting to monitor the temporal evolution of the degree distribution. As proposed by Moore, Ghoshal, and Newman (see [20]), the number of nodes with degree $k$ at time $t+\Delta$ can be written as

$$
\begin{aligned}
N(t) p_{k}(t+\Delta)= & N(t) p_{k}(t)+N(t) \mu \Delta\left[-p_{k}(t)-k p_{k}(t)\right. \\
& +(k+1) p_{k+1}(t)+\vartheta \pi_{k-1} p_{k-1}(t) \\
& \left.-\vartheta \pi_{k} p_{k}(t)+\phi_{k}\right],
\end{aligned}
$$

where $\phi_{k}$ is the probability that a newborn node has degree $k, \vartheta=\sum_{k} k \phi_{k}$ is the average degree of newborn nodes, and $\pi_{k}$ is proportional to the probability that a newborn node links to an existing degree- $k$ node. The specific functional forms of the natality profile $\phi_{k}$ and of the attachment profile $\pi_{k}$ must obey the constraints $\sum_{k} \phi_{k}=1$ and $\sum_{k} \pi_{k} p_{k}=1$, and they are detailed below for the cases of interest. Each term in (1) describes one of the possible mechanisms that alter the degree of a generic node, thus changing the entire degree distribution of the network (see [20] for details): the removal due to death $\left[-p_{k}\right]$, the passage of a node from degree $k+$ 1 to $k\left[(k+1) p_{k+1}\right]$ and from $k$ to $k-1\left[-k p_{k}\right]$ when a neighbor dies, the passage of a node from degree $k-1$ to $k$ $\left[\vartheta \pi_{k-1} p_{k-1}\right]$ and from $k$ to $k+1\left[-\vartheta \pi_{k} p_{k}\right]$ when a newborn node attaches to it, and the insertion of new nodes with degree $k\left[\phi_{k}\right]$. The equations for $k=0$ and $k=\bar{k}$ are slightly different, in an almost obvious way, and are therefore omitted. Dividing both sides of (1) by $N(t)$ and taking the limit for $\Delta \rightarrow 0$, we obtain

$$
\begin{aligned}
\dot{p}_{k}(t)= & -\mu p_{k}(t)-\mu k p_{k}(t)+\mu(k+1) p_{k+1}(t) \\
& +\mu \vartheta \pi_{k-1} p_{k-1}(t)-\mu \vartheta \pi_{k} p_{k}(t)+\mu \phi_{k} .
\end{aligned}
$$

The resulting degree distribution of the network can be obtained by integrating Eq. (2) once the functions $\phi_{k}$ and $\pi_{k}$ and the initial conditions $p_{k}(0), k=0,1, \ldots, \bar{k}$, have been specified. In other words, the long-term network topology is the attractor reached by the dynamical system (2) starting from a particularly relevant configuration. In the epidemiological context, the prototypical network structures used are the Erdös-Rényi network and the scale-free network.

\section{A. Erdös-Rényi networks}

An Erdös-Rényi network (ERN) [21] is obtained by randomly connecting $N$ nodes with a prescribed number of links. The degree distribution of an ERN with large $N$ and average degree $\langle k\rangle$ is given by a Poisson distribution (e.g., [22]),

$$
p_{k}=\frac{\exp (-\langle k\rangle)\langle k\rangle^{k}}{k !} .
$$

To analyze the effects of the birth-death process on the network structure, consider the case in which the newborn nodes are Poisson-distributed too, with a mean $\vartheta$, i.e.,

$$
\phi_{k}=\frac{\exp (-\vartheta) \vartheta^{k}}{k !} .
$$

Also, assume that each newborn individual links to existing nodes at random, independently of their degrees, i.e., $\pi_{k}=1$ for all $k$. In such a case, it can be proved [20] that the equilibrium of system (2) is a Poisson distribution. If the initial network is an ERN with distribution (3) and $\langle k\rangle=\vartheta$, the distribution remains unchanged through time. In addition to the solution provided by [20], we prove that such a fixed point of system (2) is globally asymptotically stable, and thus it is reached regardless of the initial degree distribution $p_{k}(0)$. As a matter of fact, introducing the column vectors $\mathbf{p}=$ $\left[p_{0}, p_{1}, \ldots, p_{k}, \ldots\right]^{\mathrm{T}}$ and $\boldsymbol{\Phi}=\left[\phi_{0}, \phi_{1}, \ldots, \phi_{k}, \ldots\right]^{\mathrm{T}}$, Eq. $(2)$ can be written as

$$
\dot{\mathbf{p}}(t)=\mathbf{A}(\mathbf{p}) \mathbf{p}(t)+\mu \boldsymbol{\Phi}
$$


where the matrix

$$
\mathbf{A}(\mathbf{p})=\mu\left(\begin{array}{cccccc}
-1-\vartheta \pi_{0} & 1 & 0 & 0 & 0 & \cdots \\
\vartheta \pi_{0} & -2-\vartheta \pi_{1} & 2 & 0 & 0 & \cdots \\
0 & \vartheta \pi_{1} & -3-\vartheta \pi_{2} & 3 & 0 & \cdots \\
0 & 0 & \vartheta \pi_{2} & -4-\vartheta \pi_{3} & 4 & \cdots \\
\vdots & \vdots & \vdots & \vdots & \vdots & \ddots
\end{array}\right)
$$

depends on $\mathbf{p}$, in general, because the $\pi_{k}$ 's might do so. In this specific case, however, $\pi_{k}=1$ for all $k$, so that $\mathbf{A}(\mathbf{p})=\mathbf{A}$ is constant (i.e., independent of the current network topology) and Metzler (i.e., all off-diagonal entries are non-negative). Since all column sums of $\mathbf{A}$ are negative, its dominant (Frobenius) eigenvalue is guaranteed to be negative (e.g., [23]). Therefore, Eq. (5) is a time-invariant, linear, asymptotically stable system. The unique steady-state degree distribution of (2) is reached from any initial condition.

\section{B. Scale-free networks}

Scale-free networks (SFNs) are highly heterogeneous structures, in which no node can be defined as "typical" since very few nodes (the hubs) are connected with many others, while the great majority of the nodes have only a few connections. The degree distribution of a SFN, at least for large $k$, is a power law of the form

$$
p_{k} \sim k^{-c}
$$

where $c>0$. In the past decade, SFNs have received great attention because they emerged in a variety of social and technological contexts [24-26], including epidemiology (e.g., [27]). A peculiarity in (7) is that its second moment $\left\langle k^{2}\right\rangle=\sum_{k} k^{2} p_{k}$ diverges when $N \rightarrow \infty$ if $2<c \leqslant 3$, a range of values that often appears in data [28].

Consistently with the preferential attachment paradigm proposed by Barábasi-Albert and used to generate SFNs [24],

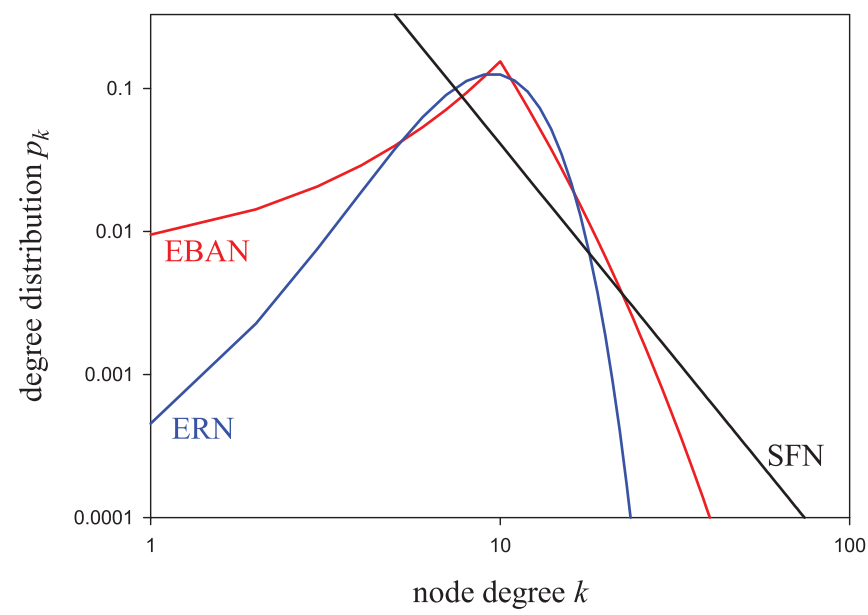

FIG. 1. (Color online) Examples of the degree distributions of an Erdös-Rényi network (ERN), a scale-free network [SFN, Eq. (7), with $c=3$ ], and an evolved Barábasi-Albert network (EBAN). All distributions have the same average degree $\langle k\rangle=10$. we assume that all newborn nodes in the network evolving on the basis of Eq. (2) have the same prescribed degree $\vartheta$ (thus $\phi_{k}=1$ for $k=\vartheta$, and 0 otherwise). Also, we imagine that newborns preferentially attach their links to nodes with high degrees $\left(\pi_{k}=k /\langle k\rangle\right)$. In this case, as proved by [20], the fixed point of (2) turns out to have a functional form that is not scale-free but "stretched exponential," namely

$$
p_{k} \sim k^{-3 / 4} e^{-2 \sqrt{k}}
$$

We call a network resulting from the process described above an evolved Barábasi-Albert network (EBAN), since the insertion of new nodes follows the standard rules proposed by [24], yet the network's evolution is driven by deaths, and not only births as in the standard Barábasi-Albert model. Note that the matrix $\mathbf{A}(\mathbf{p})$ of Eq. (5) does depend on the $p_{k}$ 's because, in this case, $\pi_{k}=k /\langle k\rangle=k /\left(\sum_{h} h p_{h}\right)$. System (5) is therefore nonlinear and, although the existence and uniqueness of the fixed point (8) have been formally proved (see [20]), the analysis of its stability is far from trivial. Nonetheless, all numerical simulations we performed indicate that the fixed-point distribution (8) is reached from all initial degree distributions, including when $p_{k}(0) \sim k^{-c}$. In other words, even if the network topology we start from is power-law and the birth (attachment) mechanism is fully compliant with the Barabási-Albert rule for creating SFNs [24], the existence of a death (detachment) process destroys the attractiveness of the scale-free distribution. Scale-free networks thus become transient states rather than attractors of system (2). Notably, in contrast to what happens with the power-law distribution, the second moment $\left\langle k^{2}\right\rangle$ of (8) remains bounded even in the theoretical limit $N \rightarrow \infty$. This means that the degree distribution of an EBAN loses its "fat-tail" [20], a feature that makes SFNs so peculiar.

For a direct and qualitative comparison, some exemplificative degree distributions of an ERN, a SFN, and an EBAN with the same average degree are depicted in Fig. 1.

\section{EPIDEMIC DYNAMICS ON VITAL NETWORKS}

We study a SIRS contact process with demography [29], which, under the standard "homogeneous mixing" hypothesis [30], is described by the following ODE model:

$$
\begin{aligned}
& \dot{s}(t)=\mu-\mu s(t)-\beta s(t) y(t)+\alpha r(t), \\
& \dot{y}(t)=\beta s(t) y(t)-(\mu+\gamma) y(t), \\
& \dot{r}(t)=\gamma y(t)-(\mu+\alpha) r(t) .
\end{aligned}
$$

Equation (9) is easily derived by normalization of the classical endemic model presented in [31]. The variables $s(t), y(t)$, and $r(t)$ represent the fraction of susceptible (or infectable), 
infected (thus infective), and recovered individuals in the population. It must be noticed that the birth and death rates are per capita and independent of epidemiological states. Also, no vertical transmission takes place (i.e., all newborns are susceptible). Notice that the first equation of system (9) is a simplification of the full equation for the susceptibles,

$$
\dot{s}(t)=\mu[s(t)+y(t)+r(t)]-\mu s(t)-\beta s(t) y(t)+\alpha r(t),
$$

obtained by using the equality $s(t)+y(t)+r(t)=1$, and that the equation for $r(t)$ is redundant since $r(t)=1-s(t)-y(t)$. Besides the birth-death rate $\mu$, the parameters appearing in (9) are the loss of immunity rate $\alpha$, the recovery rate $\gamma$, and the contact rate $\beta$, which can be interpreted as $\beta=\rho \hat{n}$, namely as the product of the disease-specific transmission rate $\rho$ and of the effective number of contacts per unit time $\hat{n}$, which is a characteristic of the population [30]. Provided that $\beta$ is not too small $(\beta>\mu+\gamma)$, it can easily be verified that system (9) has a unique endemic equilibrium with

$$
y=\frac{\alpha+\mu}{\alpha+\mu+\gamma}\left(1-\frac{\mu+\gamma}{\beta}\right) .
$$

Such an equilibrium is globally asymptotically stable [32], in other words the long-term behavior of the epidemiological process is constant. In the following, we will be mostly interested in the SIR case, obtained assuming permanent immunity $(\alpha=0)$, which is customarily used to describe a number of diseases, including childhood epidemics such as measles, rubella, or chicken pox [33].

To study the dynamics of the birth-death-infection-recovery process over a network, we follow an approach similar, yet not identical, to the one that is often used to model epidemics on networks, based on the assumption that all nodes with the same degree are statistically equivalent [4,5,28]. Our $3 d$ state variables, with $d=\bar{k}+1$, are the fraction, over the total population size $N(t)$, of individuals that have degree $k$ and are susceptible $\left[s_{k}(t)\right]$, infected $\left[y_{k}(t)\right]$, or recovered $\left[r_{k}(t)\right]$ at time $t$. Note that here $s_{k}(t)+y_{k}(t)+r_{k}(t)=p_{k}(t)$, and not unity. The equations governing the temporal evolution of the state variables incorporate the birth and death mechanisms that affect all nodes independently of their epidemiological state, as described in the preceding section. Peculiar to the epidemics are, instead, the infection of susceptibles, the recovery of the infected, and the loss of immunity of the recovered. The last two mechanisms are simple to model, since during a short time interval $\Delta$ an infected node can recover with probability $\gamma \Delta$, while a recovered node can rejoin susceptibles with probability $\alpha \Delta$. The infection mechanism is instead more complex, because a susceptible node, say $j$, can become infected with probability $\rho n_{j} \Delta$, where $n_{j}$ is the number of infected nodes among its neighbors. Neglecting degree correlations, the probability that the neighbor of a node has degree $h$ is $q_{h}=h p_{h} /\langle k\rangle$ (e.g., [26]). Since the probability that a degree- $h$ node be infected is $y_{h} / p_{h}$, the expected number of infected neighbors of a degree- $k$ node is

$$
e_{k}=k \sum_{h} \frac{h p_{h}}{\langle k\rangle} \frac{y_{h}}{p_{h}} .
$$

Therefore, the number of susceptible nodes of degree $k$ that become infected during $\Delta$ is $\rho e_{k} N s_{k} \Delta$. By combining demography (Sec. II) and epidemiological dynamics, we finally obtain the following system of $3 d$ equations:

$$
\begin{aligned}
\dot{s}_{k}(t)= & -\mu s_{k}(t)-\mu k s_{k}(t)+\mu(k+1) s_{k+1}(t) \\
& +\mu \vartheta \pi_{k-1} s_{k-1}(t)-\mu \vartheta \pi_{k} s_{k}(t)+\mu \phi_{k} \\
& -\rho k s_{k}(t) \sum_{h} \frac{h}{\langle k\rangle} y_{h}(t)+\alpha r_{k}(t), \\
\dot{y}_{k}(t)= & -\mu y_{k}(t)-\mu k y_{k}(t)+\mu(k+1) y_{k+1}(t) \\
& +\mu \vartheta \pi_{k-1} y_{k-1}(t)-\mu \vartheta \pi_{k} y_{k}(t) \\
& +\rho k s_{k}(t) \sum_{h} \frac{h}{\langle k\rangle} y_{h}(t)-\gamma y_{k}(t), \\
\dot{r}_{k}(t)= & -\mu r_{k}(t)-\mu k r_{k}(t)+\mu(k+1) r_{k+1}(t) \\
& +\mu \vartheta \pi_{k-1} r_{k-1}(t)-\mu \vartheta \pi_{k} r_{k}(t)+\gamma y_{k}(t)-\alpha r_{k}(t) .
\end{aligned}
$$

Note that the contact rate $\beta=\rho \hat{n}$ of the homogeneous mixing SIRS model (9) is now replaced by a degree-dependent contact rate $\beta_{k}=\rho k$. The global disease prevalence $y(t)$, namely the total fraction of infected in the population, is given by

$$
y(t)=\sum_{k} y_{k}(t)
$$

It is straightforward to check that the fundamental Eq. (2) is easily obtained by summing up all $3 d$ equations (13) for a fixed $k$. Since $s_{k}+y_{k}+r_{k}$ is not constant over time, we cannot simplify the study of (13) by eliminating the variables $r_{k}$ and their equations. However, being the birth-death process uncoupled from the infection-recovery process, we can ask if and how the disease spreads in a population that has already reached its demographic equilibrium. Assuming that Eq. (2) has reached its steady state, we reduce system (13) by focusing only on the dynamics of $s_{k}$ and $y_{k}$, since at any time $r_{k}=p_{k}-$ $s_{k}-y_{k}$. We note that, although it would be interesting to obtain the SIRS model (9) as a particular case of our model (13), this is not possible mathematically. Such impossibility can easily be understood from the epidemiological perspective, because the random nature of contacts in the homogeneous mixing approach cannot be reduced to the permanency of the links in the network model [30].

Let us now focus our attention to the SIR case ( $\alpha=0$ ), for which infected individuals get permanent immunity when recovered. The steady-state behavior of system (13) is summarized in Fig. 2. The first remarkable consequence of accounting for vital dynamics in the epidemic process is the existence of a finite epidemic threshold even in the EBAN case, an effect of the loss of the fat tail in the degree distribution. As already evidenced by Piccardi and Casagrandi for SIS processes [17], this outcome sharply contrasts the well-known findings of Pastor-Satorras and Vespignani [4,5] (see also [34] on this subject). The prevalence $y$ in Fig. 2, which becomes nonzero above suitable thresholds of $\rho$ and/or $\langle k\rangle$, monotonically increases with both quantities, hence with $\left\langle\beta_{k}\right\rangle=\rho\langle k\rangle$. This is qualitatively consistent with the homogeneous mixing model (9), as made clear from Eq. (11). However, a closer analysis reveals that the two models display non-negligible quantitative differences at low transmission 

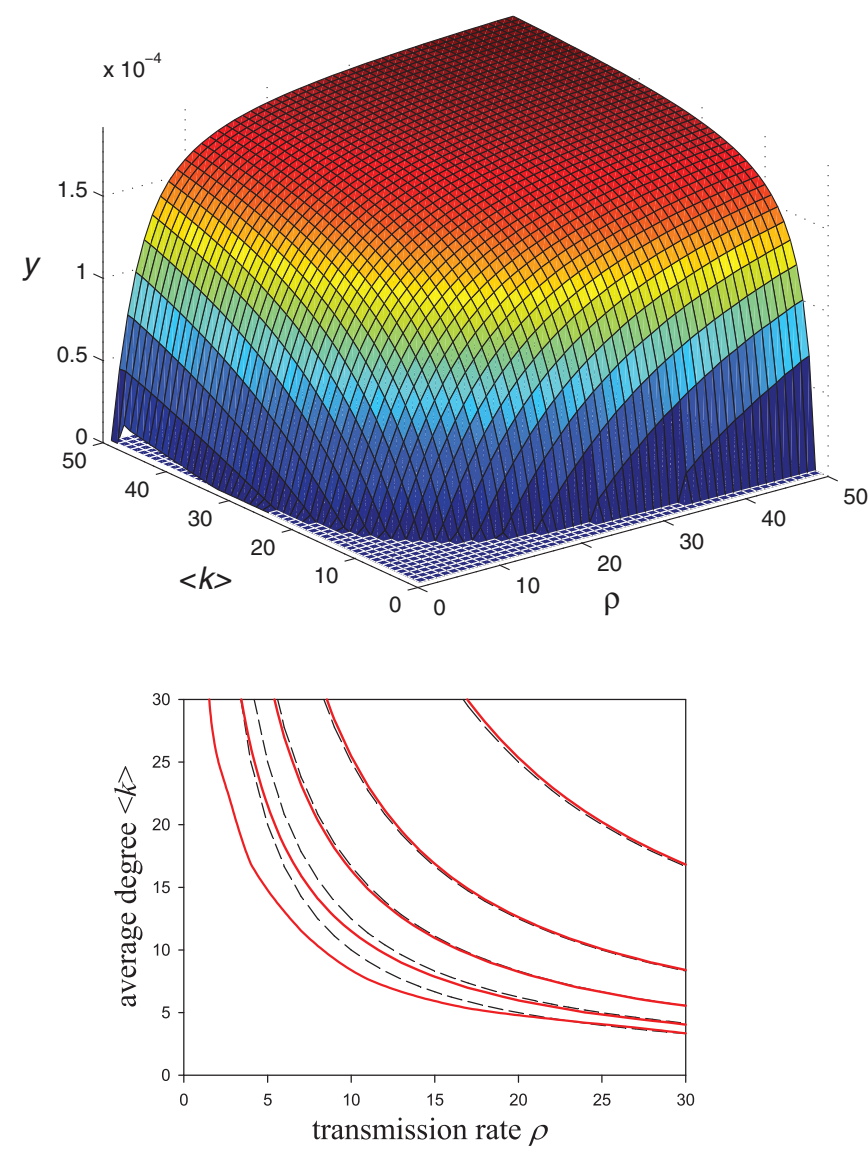

FIG. 2. (Color online) The equilibrium value of the disease prevalence $y$ on the network model (13) for an SIR model $(\alpha=0)$ in the case of EBAN, as a function of the transmission rate $\rho$ and of the average degree $\langle k\rangle$ (similar results—not shown-are obtained with ERN). In the bottom panel, the contour lines of $y=y(\rho,\langle k\rangle)$ (solid red lines) obtained by model (13) are contrasted to the iso- $\beta$ lines $\rho\langle k\rangle=$ const (dashed black lines), which are the iso- $y$ lines of the homogeneous mixing model (9). Parameter values $(\mu=0.02$, $\gamma=100)$ fall in the range that [33] considers consistent with measles dynamics.

rates $\rho$, where the network model systematically predicts that the threshold values for $\langle k\rangle$ under which the disease is eradicated be lower than those for the homogeneous mixing model (9). Also, above such thresholds, the prevalence $y$ is always larger in the network model. Overall, the network model predicts quite a stronger capability of the infection, if compared to the homogeneous mixing model, and a better ability of sustaining and propagating diseases characterized by small contact rates $\beta$.

The most interesting results are obtained from network model (13) when, instead of computing global quantities such as the prevalence $y$, we analyze how the disease is distributed among nodes with different characteristics. Figure 3 shows that, as one may expect, the distribution of the infected $y_{k}$ through the nodes with different degree $k$ essentially (and not surprisingly) replicates the degree distribution $p_{k}$. However, if we compute the proportion of infected $y_{k} / p_{k}$, that is, the probability that a degree- $k$ node will be infected, the result is quite unexpected. For ERNs, $y_{k} / p_{k}$ monotonically increases up to a plateau level, meaning that the nodes with large degree are the main carrier of the disease and thus the main reason for its propagation. This result is fully consistent with the well-known findings on SIS epidemics propagating in networks with no demography $[4,5]$.

The epidemiological scenario of EBANs is, however, completely different from that of ERNs, despite the qualitative similarity of their degree distributions (see again Fig. 1): in EBANs, not only the infected $y_{k}$, but also their proportion $y_{k} / p_{k}$, follow a distribution that qualitatively replicates the degree distribution $p_{k}$ (see Fig. 3). In other words, if we group the individuals by their number of contacts $k$, the probability of finding infected individuals is largest in the most represented set. On the contrary, such a probability tends to zero for larger and larger values of $k$. This means that, contrary to a widespread belief regarding both human (e.g., $[6,7,35,36]$ ) and animal diseases (e.g., [37]), the most connected nodes are not necessarily at highest risk of infection when demography is stretching the network topology, even if the original contacts followed a scale-free distribution. This may obviously have implications in the design of effective prevention policies.

It is interesting to discuss if - and to what extent-the above result depends on the specific assumptions used to describe the demographic evolution of the network. In Fig. 3, we used two prototypical models: ERN, where the natality profile $\phi_{k}$ is Poisson and the attachment profile $\pi_{k}$ is uniform (or "flat," i.e., $\pi_{k}=1$ for all $k$ ), and EBAN, where $\phi_{k}$ is a singleton (i.e., a spike distribution at $k=\theta$ ) and $\pi_{k}$ is "preferential" (in the manner of Barabási-Albert). We can easily swap the assumptions and generate, for example, two types of "hybrid" networks, one with Poisson natality and preferential attachment and another with singleton natality and flat attachment. The two degree distributions are obtained by letting the demographic system (2) evolve, from feasible initial conditions, until the unique equilibrium distribution is reached. The dynamics of the epidemics on such networks is then obtained by model (13). As displayed in Fig. 4 [panels (a) and (b)], the results are consistent with those of the EBAN above, with the largest proportion of infected $y_{k} / p_{k}$ at intermediate degrees, where the degree distribution of the individuals also peaks, independent of their epidemiological state. Since Eq. (2) allows for highly flexible definitions of $\phi_{k}$ and $\pi_{k}$, we can also test less idealized assumptions. For example, we can consider a Poisson natality with a truncated tail (i.e., we let $\phi_{k}=0$ for all $k$ larger than a prescribed value, renormalizing $\phi_{k}$ to have unit sum) to avoid unrealistically large degrees of newborns. Again, the result is qualitatively the same [panel (c) of Fig. 4]. Therefore, our result that the largest probability of infection is for nodes at intermediate degrees seems to be quite general for the SIR epidemic process, with the ERN case being the exception.

A different conclusion is obtained if, instead of considering diseases that confer lifelong immunity, we analyze SIRS processes $(\alpha>0)$. This mechanism to yield susceptible individuals - qualitatively different from birth - produces, in a sense, dominant effects over those induced by the previously discussed underlying demographic dynamics. Such epidemics becomes, in many respects, similar to those that would be obtained with an SIS without demography, as in fact we recover in that case the well-known behavior evidenced by [4,5], 

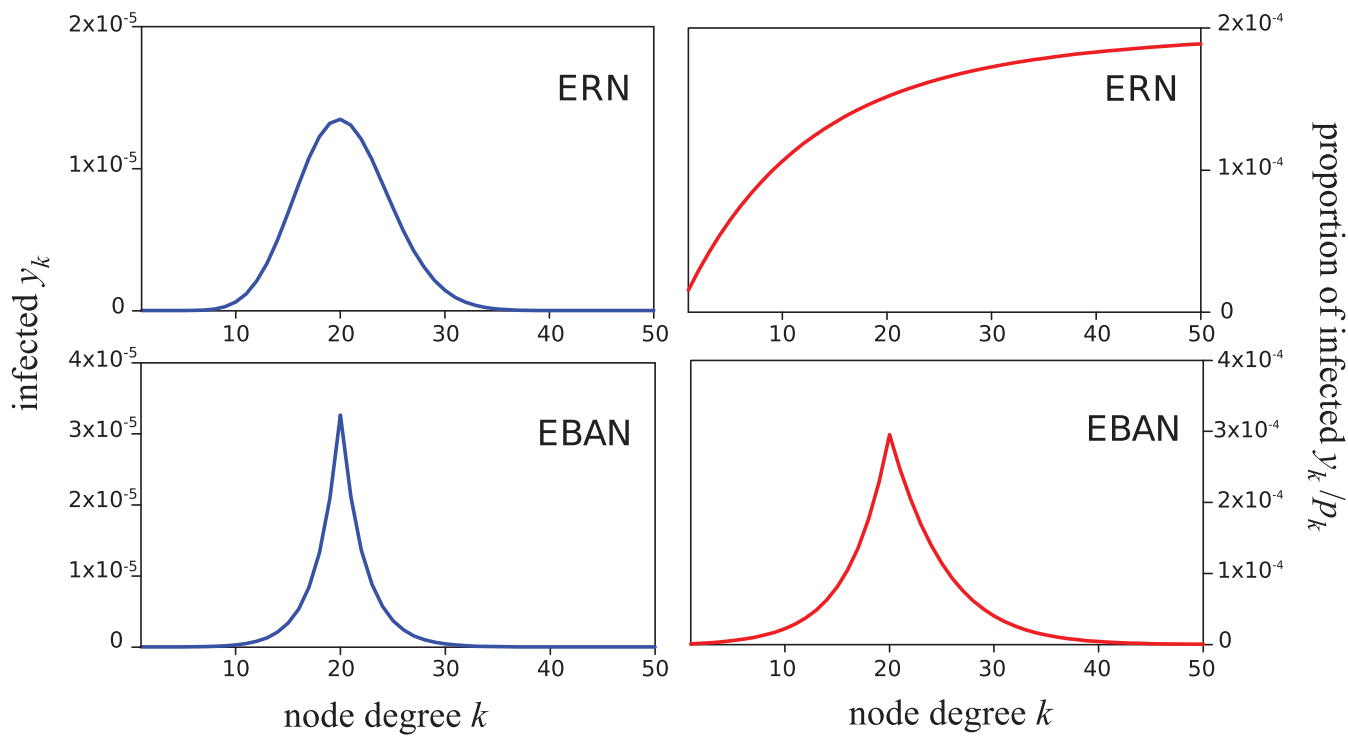

FIG. 3. (Color online) The infected $y_{k}$ (left column) and the proportion of infected $y_{k} / p_{k}$ (right column) in SIR model ( $\left.\alpha=0\right)$, as a function of the node degree $k$, for ERN (upper panels) and EBAN (lower panels). Parameter values are $\rho=20,\langle k\rangle=20, \mu=0.02, \gamma=100$.

where the larger the degree of a node is, the higher is its probability of being infected [Fig. 4(d)]. The role of vital demography, however, makes our case more involved. SIR and SIRS processes fundamentally differ in the relationship between the infected state of individuals and their age. Indeed, in the SIR process, the eldest individuals tend to accumulate in the compartment of the recovered, whereas this has no reason to occur if immunity is lost during life as in the SIRS process. To explore in detail the implications of such subtle but crucial differences, and to investigate why the ERN case is so peculiar, we explicitly account below for the age of individuals in our mathematical model.

\section{AN EPIDEMIC MODEL WITH AGE AND DEGREE STRUCTURE}

We generalize the description of the disease dynamics on vital networks so that at any time $t$ each individual (node) is characterized by (i) its degree $k$, (ii) its epidemiological state (susceptible, infected, or recovered), and (iii) its age $a$, defined as the time passed since the node was added to the network. We denote by $S_{k}(a, t), Y_{k}(a, t)$, and $R_{k}(a, t)$ the distributions of, respectively, susceptibles, infected, and recovered that at time $t$ have age $a$ and degree $k$. For any $k, t$, and $a$, we obtain the age distribution of degree- $k$ nodes $P_{k}(a, t)$ as equal to $S_{k}(a, t)+$
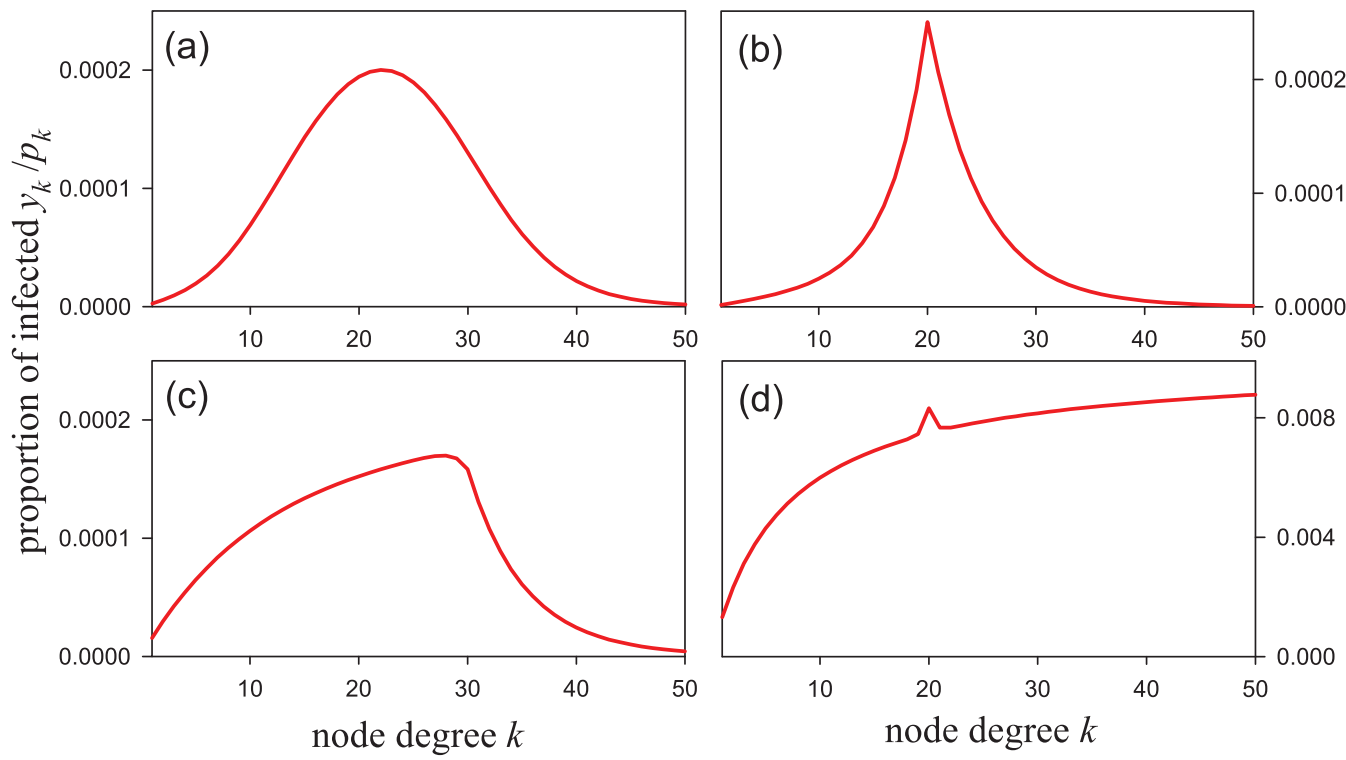

FIG. 4. (Color online) The proportion of infected $y_{k} / p_{k}$ obtained with model (13) as a function of the node degree $k$, for a few networks with mixed features. (a) SIR process $(\alpha=0)$ : Poisson natality, preferential attachment. (b) SIR process: Singleton natality, flat attachment. (c) SIR process: Poisson natality truncated at $k=30$, flat attachment. (d) SIRS process $(\alpha=10)$ : EBAN (singleton natality, preferential attachment). Other parameter values are $\rho=20,\langle k\rangle=20, \mu=0.02, \gamma=100$. 
$Y_{k}(a, t)+R_{k}(a, t)$. These newly introduced quantities relate to the (age-independent) ones formerly used in Eqs. (2) and (13) as follows:

$$
\begin{aligned}
& s_{k}(t)=\int_{0}^{+\infty} S_{k}(a, t) d a, \quad y_{k}(t)=\int_{0}^{+\infty} Y_{k}(a, t) d a, \\
& r_{k}(t)=\int_{0}^{+\infty} R_{k}(a, t) d a, \quad p_{k}(t)=\int_{0}^{+\infty} P_{k}(a, t) d a .
\end{aligned}
$$

The equations governing the dynamics of the newly introduced variables can be obtained with the standard procedure used to derive, in population dynamics, age-structured models in continuous time [38]. Since $t$ and $a$ grow at the same rate, the basic balance law for, e.g., degree- $k$ susceptibles takes the form

$$
S_{k}(a+\Delta, t+\Delta)=S_{k}(a, t)+(\text { inflow }- \text { outflow }) \Delta,
$$

where the inflow and outflow terms account for all the mechanisms that alter either the degree or the epidemic state of a node, as evidenced in Eqs. (2) and (13). Letting $\Delta \rightarrow 0$, we obtain the following system of partial differential equations:

$$
\begin{aligned}
& \frac{\partial S_{k}(a, t)}{\partial a}+\frac{\partial S_{k}(a, t)}{\partial t} \\
& =-\mu S_{k}(a, t)-\mu k S_{k}(a, t)+\mu(k+1) S_{k+1}(a, t) \\
& \quad+\mu \vartheta \pi_{k-1} S_{k-1}(a, t)-\mu \vartheta \pi_{k} S_{k}(a, t) \\
& \quad-\rho k S_{k}(a, t) \sum_{h} \frac{h}{\langle k\rangle} y_{h}(t)+\alpha R_{k}(a, t), \\
& \frac{\partial Y_{k}(a, t)}{\partial a}+\frac{\partial Y_{k}(a, t)}{\partial t} \\
& \quad-\mu Y_{k}(a, t)-\mu k Y_{k}(a, t)+\mu(k+1) Y_{k+1}(a, t) \\
& \quad+\mu \vartheta \pi_{k-1} Y_{k-1}(a, t)-\mu \vartheta \pi_{k} Y_{k}(a, t) \\
& \quad+\rho k S_{k}(a, t) \sum_{h} \frac{h}{\langle k\rangle} y_{h}(t)-\gamma Y_{k}(a, t), \\
& \frac{\partial R_{k}(a, t)}{\partial a}+\frac{\partial R_{k}(a, t)}{\partial t} \\
& \quad+\mu R_{k}(a, t)-\mu k R_{k}(a, t)+\mu(k+1) R_{k+1}(a, t) \\
& \quad+\mu \vartheta \pi_{k-1} R_{k-1}(a, t)-\mu \vartheta \pi_{k} R_{k}(a, t) \\
& \quad+\gamma Y_{k}(a, t)-\alpha R_{k}(a, t) .
\end{aligned}
$$

Note that the flow of new infecteds $\rho k S_{k}(a, t) \sum_{h}(h /\langle k\rangle) y_{h}(t)$ depends on the $y_{h}$, i.e., the age-independent distribution of infecteds over the node degrees. Indeed, the probability that a susceptible with age $a$ will become infected is obviously independent of the age of the infected with whom she comes into contact. Because of this specific term, the above model is actually an integrodifferential model, with $y_{h}$ depending on $Y_{h}(a, t)$ as specified in (15). In the case of no vertical transmission, i.e., when all newborns are susceptibles, a plausible set of boundary conditions is

$$
S_{k}(0, t)=\mu \phi_{k}, \quad Y_{k}(0, t)=0, \quad R_{k}(0, t)=0, \quad \forall t \geqslant 0 .
$$

Model (17) describes the spread of a SIRS process over a network with vital demography, and it accounts both for the number of contacts of individuals and for their age. The model could be refined along many directions. The biological parameters $\alpha, \gamma$, and $\rho$ could, for example, be made age-dependent. The same holds for the mortality $\mu$, with the only caveat being to constrain its mean value to equal the natality rate in order the keep the population constant in demographic terms.

Here our main goal, however, is to gain insight into the solutions of the age-independent model (13) discussed in the preceding section. For that, we will restrict our attention to steady-state solutions. In such conditions, we first derive the distribution of infected $y_{k}$ from (13) and plug it into (17). Being at equilibrium, time derivatives are nullified. We obtain the following system of ordinary differential equations:

$$
\begin{aligned}
\frac{d S_{k}(a)}{d a}= & -\mu S_{k}(a)-\mu k S_{k}(a)+\mu(k+1) S_{k+1}(a) \\
& +\mu \vartheta \pi_{k-1} S_{k-1}(a)-\mu \vartheta \pi_{k} S_{k}(a) \\
& -\rho k S_{k}(a) \sum_{h} \frac{h}{\langle k\rangle} y_{h}+\alpha R_{k}(a), \\
\frac{d Y_{k}(a)}{d a}= & -\mu Y_{k}(a)-\mu k Y_{k}(a)+\mu(k+1) Y_{k+1}(a) \\
& +\mu \vartheta \pi_{k-1} Y_{k-1}(a)-\mu \vartheta \pi_{k} Y_{k}(a) \\
& +\rho k S_{k}(a) \sum_{h} \frac{h}{\langle k\rangle} y_{h}-\gamma Y_{k}(a), \\
\frac{d R_{k}(a)}{d a}= & -\mu R_{k}(a)-\mu k R_{k}(a)+\mu(k+1) R_{k+1}(a) \\
& +\mu \vartheta \pi_{k-1} R_{k-1}(a)-\mu \vartheta \pi_{k} R_{k}(a) \\
& +\gamma Y_{k}(a)-\alpha R_{k}(a) .
\end{aligned}
$$

Solving Eq. (19) with initial conditions $S_{k}(0)=\mu \phi_{k}, Y_{k}(0)=$ $0, R_{k}(0)=0$ gives the steady-state distribution profiles $S_{k}(a)$, $Y_{k}(a)$, and $R_{k}(a)$ of degree- $k$ susceptibles, infected, and recovered, as a function of age $a$. A representative visualization of a prototypical and exemplificative solution for the SIR process $(\alpha=0)$ is displayed in Fig. 5, where the age distribution $Y_{k}(a)$

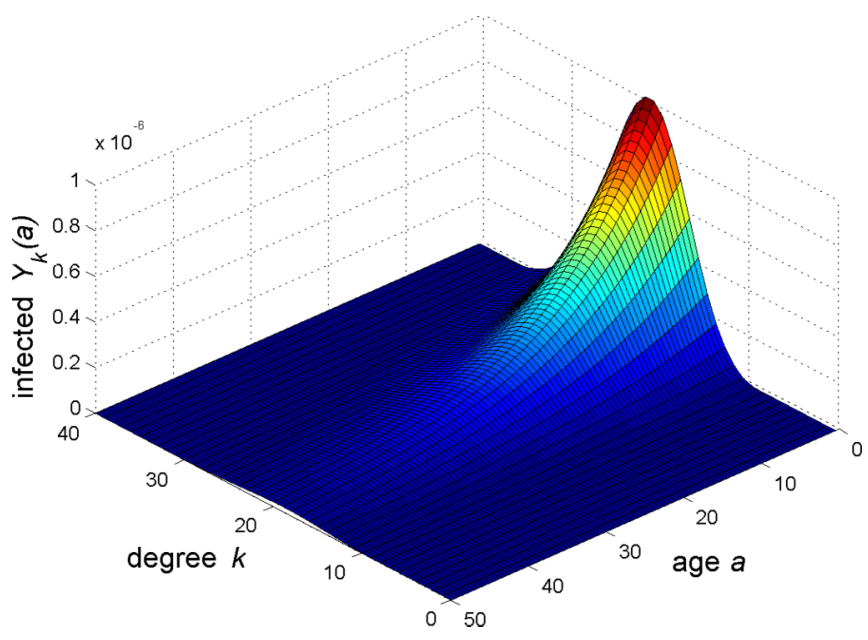

FIG. 5. (Color online) The steady-state distribution of infected $Y_{k}(a)$ as a function of age $a$ and degree $k$ of individuals, for the SIR process on ERN. Parameter values: $\rho=20,\langle k\rangle=20, \mu=0.02$, $\gamma=100$. 


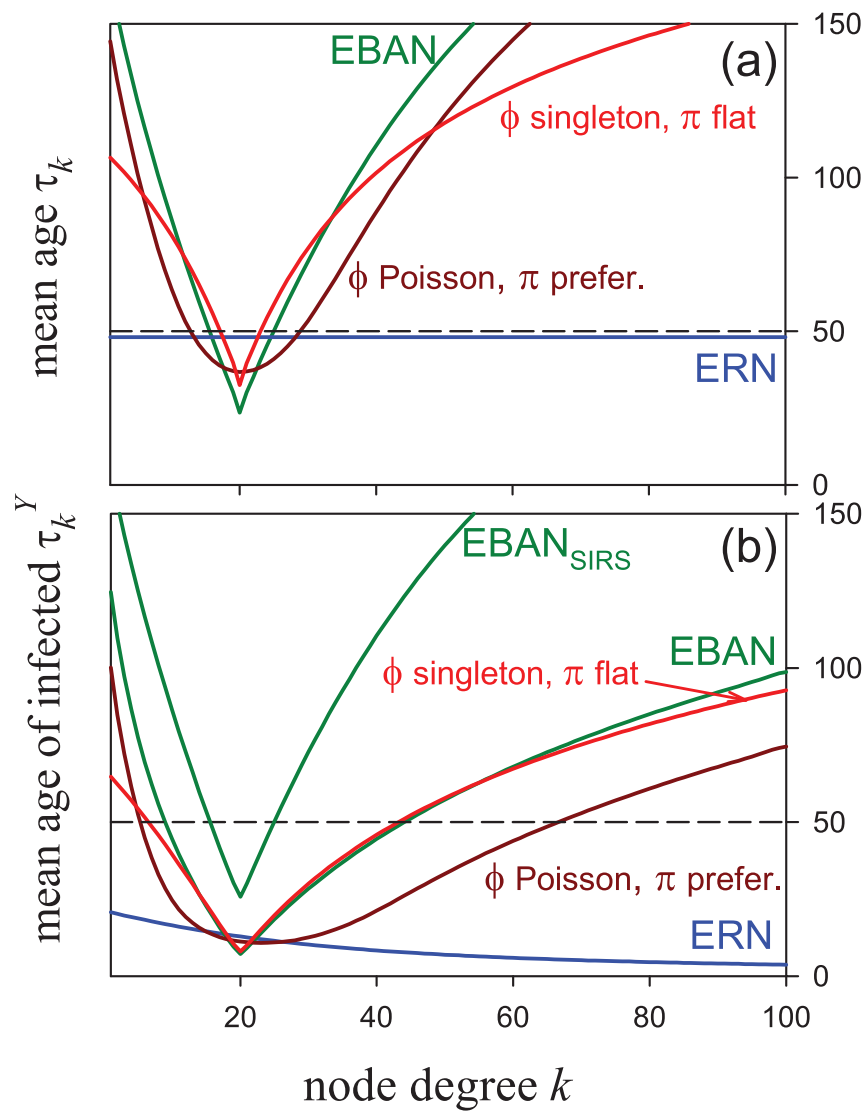

FIG. 6. (Color online) The mean age (a) of the entire population $\tau_{k}$ and (b) of the infected $\tau_{k}^{Y}$ as a function of the node degree $k$. All curves refer to the SIR process $(\alpha=0)$ except EBAN $\mathrm{EIRS}_{\text {, for }}$ which $\alpha=10$. The horizontal dashed line is the average lifetime $1 / \mu$. Parameter values: $\rho=20,\langle k\rangle=20, \mu=0.02, \gamma=100$.

of infecteds is plotted in a range of the degree $k$. For all degrees, the marginal distribution of infecteds with respect to age is monotonically decreasing. The maximal risk of infection is at birth.

Furthermore, a few measures better complement our understanding and lend themselves an insightful interpretation of the results. In Fig. 6, panel (a) shows the mean age distribution $\tau_{k}$ of the population, that is, the mean age of all individuals at the demographic equilibrium as a function of their degree $k$ :

$$
\tau_{k}=\frac{1}{p_{k}} \int_{0}^{+\infty} a P_{k}(a) d a,
$$

where $P_{k}(a)=S_{k}(a)+Y_{k}(a)+R_{k}(a)$. Notice that $\tau_{k}$ only depends on the vital demography (i.e., on the natality and attachment profiles), and not on the peculiarities of the epidemic process. It turns out that, in all cases but one, the highly connected individuals (i.e., nodes with large $k$ ) are old individuals too, namely their mean age is largely above average. ERN is the only exception, with a flat $\tau_{k}$ at the level of the average lifetime $1 / \mu$. Thus, only the combination of the assumptions of Poisson natality and flat attachment yields such a singular case. Such a peculiarity becomes even more pronounced if we consider the mean age distribution of infected $\tau_{k}^{Y}$ :

$$
\tau_{k}^{Y}=\frac{1}{y_{k}} \int_{0}^{+\infty} a Y_{k}(a) d a .
$$

In Fig. 6, panel (b) reveals that, in the ERN case, infected nodes with high connectivity (i.e., large $k$ ) are even younger that average, whereas in all other cases they are older [similarly to the entire population, as shown in panel (a)].

Another set of quantities that can be derived from (19) concerns the distribution of age profiles for the fractions of susceptibles, infected, and recovered:

$$
\begin{aligned}
& \bar{S}(a)=\frac{\sum_{k} S_{k}(a)}{\sum_{k} P_{k}(a)}, \quad \bar{Y}(a)=\frac{\sum_{k} Y_{k}(a)}{\sum_{k} P_{k}(a)}, \\
& \bar{R}(a)=\frac{\sum_{k} R_{k}(a)}{\sum_{k} P_{k}(a)}
\end{aligned}
$$

Note that $\bar{S}(a)+\bar{Y}(a)+\bar{R}(a)=1$ for all $a$. Figure 7 (a) shows that, for the SIR process, the fraction $\bar{Y}(a)$ of infecteds decays to zero as age $a$ increases. Therefore, there are practically no infecteds among the oldest part of the population, which, on the other hand, contains the most connected individuals, as discussed above. This explains the result highlighted in the preceding section, i.e., the vanishing proportion of infected $y_{k} / p_{k}$ among the most connected nodes. As already pointed out, the only exception is the ERN case in which all age classes are represented among the set of nodes with large degree $k$, which thus [see again Fig. 7(a)] contains a nonnegligible fraction of infected. Finally, if we consider the SIRS process $(\alpha>0)$, we immediately note from curve EBAN SIRS in Fig. 7(b) that the loss of immunity has the effect that the infecteds spread over all age classes and, consequently, over all degree classes as well. For that reason, we find a nonvanishing

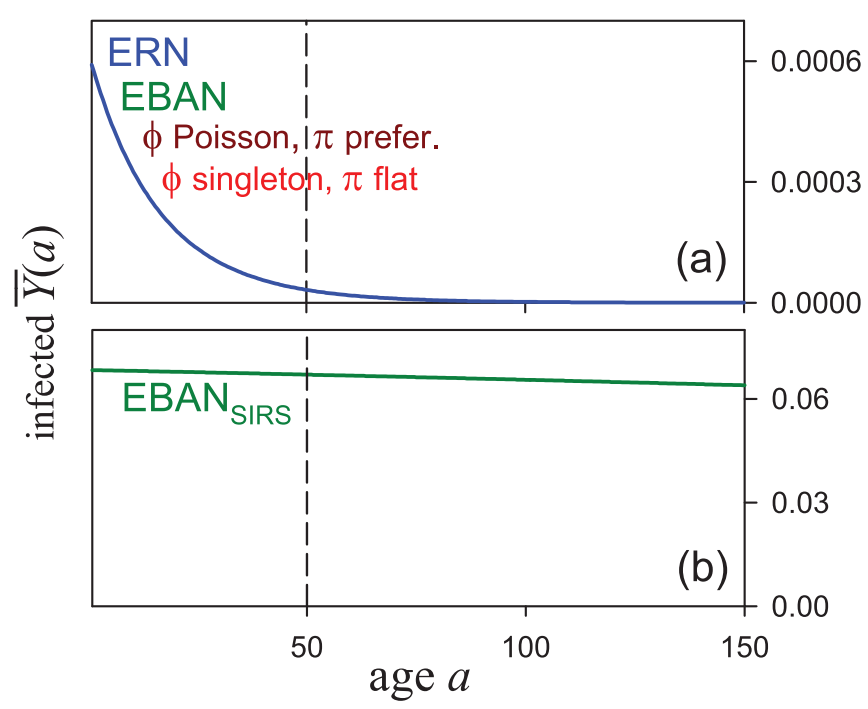

FIG. 7. (Color online) The fraction $\bar{Y}(a)$ of infected as a function of age $a$. Panel (a) refers to the SIR process $(\alpha=0)$ (the four curves are visually indistinguishable because practically coincident); panel (b) refers to the SIRS process $(\alpha=10)$ on EBAN. The vertical dashed line is the average lifetime $1 / \mu$. Parameter values: $\rho=20$, $\langle k\rangle=20, \mu=0.02, \gamma=100$. 


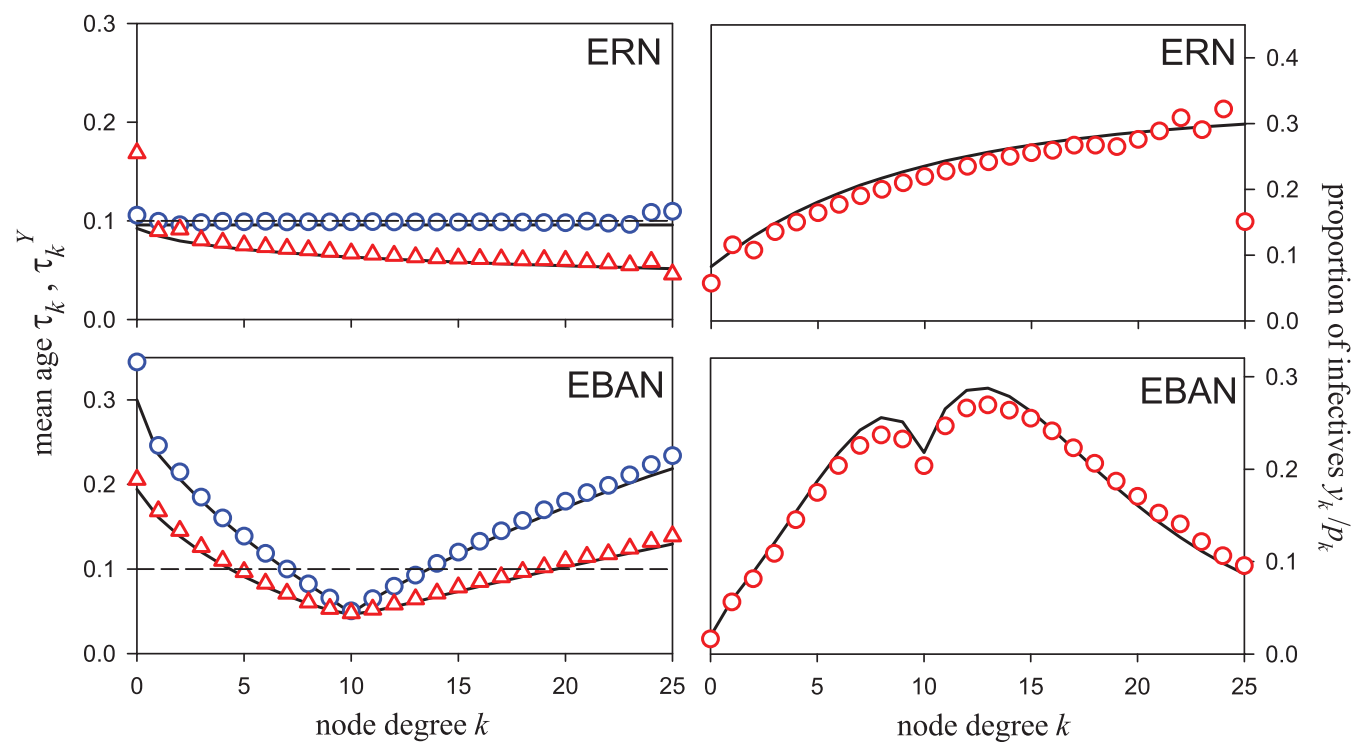

FIG. 8. (Color online) A sample of the results of the simulations of the SIR process with individual-based probabilistic cellular automata, on ERN (top panels) and EBAN (bottom panels). Left panels: the mean age of the entire population $\tau_{k}$ (blue circles) and of the infected $\tau_{k}^{Y}$ (red triangles) as a function of the node degree $k$. Right panels: the proportion of infected $y_{k} / p_{k}$ as a function of the node degree $k$. In all panels, the solid black lines are the outcome of the differential equation models (13) and (19). In the left panels, the horizontal dashed line is the average lifetime $1 / \mu$.

fraction $y_{k} / p_{k}$ of infected at large degrees $k$ in this case too, as evidenced in the preceding section.

We finally mention that, in order to rule out possible artifacts induced by approximating the time evolution of networks and epidemic processes by differential equations (13) and (19), the above findings have been validated by means of individualbased simulations based on probabilistic cellular automata. All the above-defined network configurations (i.e., natality and attachment kernels) have been considered, and SIR and SIRS epidemic processes have been simulated on networks of different size. A sample of the results, which highlights some of the most peculiar results discussed in the paper, is reported in Fig. 8 [39]. In all instances, we found very good agreement, both qualitative and quantitative, between the results of the simulations on probabilistic cellular automata and the output of the differential equation models.

\section{CONCLUDING REMARKS}

Incorporating birth and death processes into simple infection mechanisms (of both SIR and SIRS types) over homogeneous and heterogeneous networks can qualitatively change the epidemiological outcomes. As Moore and coauthors [20] have shown, demographic dynamics alone destroys the degree distribution structure in scale-free networks, even if the newborn nodes are added as in the Barabási-Albert algorithm (i.e., preferential attachment of links) and the dying nodes are detached at random. The emerging distribution [here called the evolved Barábasi-Albert network (EBAN)] is not fat-tailed anymore, so not surprisingly we find that SIR and SIRS processes can persist if and only if their contact rates are above a finite threshold. Less evident is the fact that the fraction of infected individuals in SIR processes over vital networks does not necessarily grow with the node degree-as is the case for SIS processes with or without demography on networks. For SIR processes, that peculiar result occurs only in the case of ERNs, i.e., Erdös-Rényi networks subject to Poisson births and homogeneous (i.e., degree-independent) attachment. In all other cases that we analyzed, such a proportion of infected nodes peaks at intermediate node degrees, exactly where the degree distribution of the total population does. The mechanism of immunity loss (SIRS instead of SIR process) changes the picture completely because, independent of the network structure, the fraction of infecteds increases monotonically with the node degree.

To understand why qualitatively different results are obtained by two apparently similar mechanisms of feeding the network with susceptible individuals (i.e., birth or immunity loss), we developed a model that also accounts for the age of the nodes (i.e., the time since their first appearance in the network). The age distributions of network individuals in different epidemiological states reveal that susceptibles in SIR models can only be the youngest, i.e., those who have never been in contact with the disease. In contrast, if a loss of immunity is accounted for (as in SIRS models), susceptible individuals can have entered the population either via birth (for younger nodes) or via a complete loss of immunity (for recovered nodes that were infected even long before). These two different kinds of susceptible individuals are characterized by different degrees and are not interchangeable in terms of epidemic spread. As a consequence, control strategies for SIR epidemics that are mainly based on degree distributions can fail, despite their proven efficacy when applied to SIRS processes over static networks. Identifying which individuals are at maximum risk of infection is therefore dependent in an articulate manner on (i) the network structure, (ii) the epidemiological state of the individual, and (iii) the path followed by the individual to join the susceptibles compartment of the population (either by birth or by immunity loss). 
In this paper, the proposed age-degree model has been studied only to disentangle the underlying causes of the different outcomes obtained for SIR and SIRS processes. There are of course many diseases (ranging from pertussis to tuberculosis, just to restrict our attention to humans) for which the age of the hosts influences both the demographic and the epidemiological parameters. In all these cases, our age-degree model can become a very useful tool for researchers and health managers.
[1] J. Deter, K. Berthier, Y. Chaval, J. F. Cosson, S. Morand, and N. Charbonnel, Parasitology 132, 595 (2006).

[2] S. Dorjee, C. Revie, Z. Poljak, W. McNab, and J. Sanchez, Prev. Vet. Med. 112, 118 (2013).

[3] W. O. Kermack and A. G. McKendrick, Proc. R. Soc. A 115, 700 (1927).

[4] R. Pastor-Satorras and A. Vespignani, Phys. Rev. E 63, 066117 (2001).

[5] R. Pastor-Satorras and A. Vespignani, Phys. Rev. Lett. 86, 3200 (2001).

[6] R. Cohen, S. Havlin, and D. ben-Avraham, Phys. Rev. Lett. 91, 247901 (2003).

[7] R. Christley, G. Pinchbeck, R. Bowers, D. Clancy, N. French, R. Bennett, and J. Turner, Am. J. Epidemiol. 162, 1024 (2005).

[8] P. Holme and J. Saramäki, Phys. Rep. 519, 97 (2012).

[9] Adaptive Networks: Theory, Models and Applications, edited by T. Gross and H. Sayama, Understanding Complex Systems (Springer, New York, 2009).

[10] M. Li, J. Graef, L. Wang, and J. Karsai, Math. Biosci. 160, 191 (1999).

[11] B. Murphy, B. Singer, S. Anderson, and D. Kirschner, Math. Biosci. 180, 161 (2002).

[12] J. Sanz, L. Mario Floria, and Y. Moreno, Int. J. Bifurcation Chaos 22, 1250164 (2012).

[13] V. Andreasen, C. Viboud, and L. Simonsen, J. Infect. Dis. 197, 270 (2008).

[14] R. Casagrandi, L. Bolzoni, S. A. Levin, and V. Andreasen, Math. Biosci. 200, 152 (2006).

[15] F. Brauer, in Mathematical Epidemiology, edited by F. Brauer, P. van den Driessche, and J. Wu (Springer, 2008), pp. 19-80.

[16] I. Nasell, in Mathematical Approaches for Emerging and Reemerging Infectious Diseases: An Introduction, edited by C. Castillo-Chavez, S. Blower, P. Van den Driessche, D. Kirschner, and A.-A. Yakubu (Springer, 2002), pp. 199-228.

[17] C. Piccardi and R. Casagrandi, in Modelling, Estimation and Control of Networked Complex Systems, Understanding Complex Systems-Springer Complexity, edited by A. Chiuso, L. Fortuna, M. Frasca, A. Rizzo, L. Schenato, and S. Zampieri (Springer-Verlag, Berlin, 2009), pp. 77-89.
[18] J. Sanz, L. M. Floria, and Y. Moreno, Phys. Rev. E 81, 056108 (2010).

[19] G. Demirel and T. Gross, arXiv:1209.2541 [physics.soc-ph].

[20] C. Moore, G. Ghoshal, and M. E. J. Newman, Phys. Rev. E 74, 036121 (2006).

[21] P. Erdös and A. Rényi, Publ. Math. 6, 290 (1959).

[22] M. E. J. Newman, Networks: An Introduction (Oxford University Press, Oxford, 2010).

[23] L. Farina and S. Rinaldi, Positive Linear Systems, Theory and Applications (Wiley, 2000).

[24] A. L. Barabási and R. Albert, Science 286, 509 (1999).

[25] M. E. J. Newman, SIAM Rev. 45, 167 (2003).

[26] S. Boccaletti, V. Latora, Y. Moreno, M. Chavez, and D. H. Hwang, Phys. Rep. 424, 175 (2006).

[27] F. Liljeros, C. R. Edling, L. A. N. Amaral, H. E. Stanley, and Y. Aberg, Nature (London) 411, 907 (2001).

[28] A. Barrat, M. Barthélemy, and A. Vespignani, Dynamical Processes on Complex Networks (Cambridge University Press, Cambridge, 2008).

[29] R. M. Anderson and R. M. May, Infectious Diseases of Humans, Dynamics and Control (Oxford University Press, Oxford, UK, 1992).

[30] M. J. Keeling and K. T. D. Eames, J. R. Soc. Int. 2, 295 (2005).

[31] H. Hethcote, SIAM Rev. 42, 599 (2000).

[32] M. Y. Li and J. S. Muldowney, Math. Biosci. 125, 155 (1995).

[33] L. F. Olsen, G. L. Truty, and W. M. Schaffer, Theor. Popul. Biol. 33, 344 (1988).

[34] C. Piccardi and R. Casagrandi, Phys. Rev. E 77, 026113 (2008).

[35] R. Pastor-Satorras and A. Vespignani, Phys. Rev. E 65, 036104 (2002).

[36] N. A. Christakis and J. H. Fowler, PLoS One 5, e12948 (2010).

[37] G. Fournié, J. Guitian, S. Desvaux, V. C. Cuong, D. H. Dung, D. U. Pfeiffer, P. Mangtani, and A. C. Ghani, Proc. Natl. Acad. Sci. (USA) 110, 9177 (2013).

[38] F. Hoppensteadt, Mathematical Theories of Populations, Demographics, Genetics and Epidemics (SIAM, Philadelphia, 1975).

[39] See Supplemental Material at http://link.aps.org/supplemental/ 10.1103/PhysRevE.91.022809 for the full set of experiments, with a detailed description of the simulation procedure. 